\title{
In silico analysis excavates potential biomarkers by constructing miRNA-mRNA networks between non-cirrhotic HCC and cirrhotic HCC
}

Bisha Ding ${ }^{1 \dagger}$, Weiyang Lou ${ }^{1 \dagger}$, Jingxing $\mathrm{Liu}^{2+}$, Ruohan $\mathrm{Li}^{3}$, Jing Chen ${ }^{4^{*}}$ and Weimin Fan ${ }^{{ }^{*}}$ (C)

\begin{abstract}
Background: Mounting evidences have demonstrated that HCC patients with or without cirrhosis possess different clinical characteristics, tumor development and prognosis. However, few studies directly investigated the underlying molecular mechanisms between non-cirrhotic HCC and cirrhotic HCC.

Methods: The clinical information and RNA-seq data were downloaded from The Cancer Genome Atlas (TCGA) database. Differentially expressed genes (DEGs) of HCC with or without cirrhosis were obtained by R software. Functional annotation and pathway enrichment analysis were performed by Enrichr. Protein-protein interaction (PPI) network was established through STRING and mapped to Cytoscape to identify hub genes. MicroRNAs were predicted through miRDB database. Furthermore, correlation analysis between selected genes and miRNAs were conducted via starBase database. MiRNAs expression levels between HCC with or without cirrhosis and corresponding normal liver tissues were further validated through GEO datasets. Finally, expression levels of key miRNAs and target genes were validated through qRT-PCR.
\end{abstract}

Results: Between 132 non-cirrhotic HCC and 79 cirrhotic HCC in TCGA, 768 DEGs were acquired, mainly involved in neuroactive ligand-receptor interaction pathway. According to the result from gene expression analysis in TCGA, CCL19, CCL25, CNR1, PF4 and PPBP were renamed as key genes and selected for further investigation. Survival analysis indicated that upregulated CNR1 correlated with worse OS in cirrhotic HCC. Furthermore, ROC analysis revealed the significant diagnostic values of PF4 and PPBP in cirrhotic HCC, and CCL19, CCL25 in non-cirrhotic HCC. Next, 517 miRNAs were predicted to target the 5 key genes. Correlation analysis confirmed that 16 of 517 miRNAs were negatively regulated the key genes. By detecting the expression levels of these key miRNAs from GEO database, we found 4 miRNAs have high research values. Finally, potential miRNA-mRNA networks were constructed based on the results of qRT-PCR.

\footnotetext{
*Correspondence: lou1350843886@126.com; 21718154@zju.edu.cn

†Bisha Ding, Weiyang Lou and Jingxing Liu contributed equally to this work

${ }^{1}$ Program of Innovative Cancer Therapeutics, Division of Hepatobiliary and Pancreatic Surgery, Department of Surgery, First Affiliated Hospital, College of Medicine, Key Laboratory of Combined Multi-Organ Transplantation, Ministry of Public Health, Key Laboratory of Organ Transplantation, Zhejiang University, Hangzhou 310003, Zhejiang Province, China ${ }^{4}$ First Affiliated Hospital of Jiaxing University,

Jiaxing 314000, Zhejiang, China

Full list of author information is available at the end of the article
} 
Conclusion: In silico analysis, we first constructed the miRNA-mRNA regulatory networks in non-cirrhotic HCC and cirrhotic HCC.

Keywords: Gene, miRNA, Non-cirrhotic, Cirrhotic, HCC

\section{Background}

Hepatocellular carcinoma (HCC) ranks the third leading cause of cancer death and the sixth most frequently diagnosed neoplasm among the world [1]. 782,500 new liver cancer patients were diagnosed and 745,500 deaths occurred worldwide in 2012, while China occupy half number of the total cases [2]. The occurrence of HCC is strongly correlated with etiological factors, including virus infection related-cirrhosis, alcohol abuse relatedcirrhosis, non-alcoholic fatty liver disease (NAFLD) related-cirrhosis [2-6]. It's widely known that HCC and cirrhosis are two common progressive fibrotic liver diseases causing death and the progression of HCC is closely related with cirrhosis, as the majority of HCC patients are accompanied with liver cirrhosis [7, 8]. However, there is still about $20 \%$ of HCC cases arising in non-cirrhotic livers, with limited study $[9,10]$. To date, more and more analyses revealed that HCC patients with or without highly cirrhotic liver have different pathogenetic backgrounds, clinical characteristics, tumor development, prognosis and surveillance indicators, also attributed to different risk factors [4, 11-15]. Although a lot of time and efforts have been placed to well understanding $\mathrm{HCC}$, the exact mechanisms about the difference and connection of non-cirrhotic HCC and cirrhotic HCC remain unclarified and require urgent consideration. Furthermore, effective treatments for $\mathrm{HCC}$ including cirrhotic $\mathrm{HCC}$ and non-cirrhotic $\mathrm{HCC}$ are unavailable [3].

Messenger RNA is a large family of RNA molecules that encode proteins, convey genetic information. MicroRNAs (miRNAs) are a class of small non-coding RNA molecules (containing about 22 nucleotides) that conversely regulate gene expression at the post-transcriptional level $[16,17]$. Gene and miRNA play significant roles in various essential tumor biological processes by constructing miRNA-mRNA networks $[18,19]$.

In this study, we aim to excavate different clinical pathological of non-cirrhotic HCC and cirrhotic HCC, identify dysregulated genes between HCC with or without cirrhotic, and build miRNA-mRNA networks by using clinical data and RNA-seq data in The Cancer Genome Atlas (TCGA) database. Based on bioinformatic analysis and experimental validation, the key miRNA-mRNA interactions in non-cirrhotic HCC and cirrhotic HCC were further validated, and provided us a new insight into the mechanisms, thus developing effective therapeutic strategies for HCC.

\section{Materials and methods}

Selecting non-cirrhotic and cirrhotic HCC patients and screening DEGs between non-cirrhotic and cirrhotic $\mathrm{HCC}$

The clinical pathological information and raw expression data were downloaded from The Cancer Genome Atlas (TCGA) database (https://cancergenome.nih.gov/). We only selected the samples which contain both clinical information and RNA-seq expression data, and subdivided these patients into two groups (non-cirrhotic HCC group and cirrhotic HCC group) in terms of the Ishak score [20]. Data were normalized and the differentially expression genes (DEGs) between two groups were both analyzed by edgeR package (http://bioconductor.org/packages/edgeR/) in $\mathrm{R}$ software. The $\mid$ fold change (FC) $\mid>2$ and $\mathrm{p}$-value $<0.05$ were set as restricted condition to identify DEGs.

\section{Gene ontology annotation and kyoto encyclopedia} of genes and genomes pathway enrichment analysis

The Enrichr database (http://amp.pharm.mssm.edu/ Enrichr/) was used to perform functional annotation and pathway enrichment analysis, including Gene Ontology annotation (GO) and kyoto encyclopedia of genes and genomes (KEGG) pathway analysis [21, 22].

\section{Construction and analysis of PPI network and miRNA-mRNA interaction networks}

The DEGs were entered into STRING database (https ://string-db.org/) to gain significant functional associations among genes [23]. The minimum required interaction score set as 0.4. Then, the string_interactions.tsv was downloaded from STRING and mapped to Cytoscape software (version 3.7.0) in order to find hub genes. In addition, expression levels analysis between HCC and corresponding normal tissues were performed to validate the selected hub genes. 5 of 15 hub genes were selected for the next research. Survival analysis was also performed to validate the 5 key genes. Besides, the target miRNAs of the 5 selected key genes were predicted via miRDB database (http://mirdb.org/miRDB/) [24, 25]. Next, we further screened the correlation between predicted miRNAs and target key genes based on starBase database (http:// starbase.sysu.edu.cn/panCancer.php) [26]. Only those 
miRNAs that negatively correlated with target genes and the $|r|$ value $>0.1, p$ value $<0.05$ were chosen for the following investigation. Then, we confirmed the expression of those key miRNAs between liver normal tissues and HCC with or without cirrhosis according to GSE10694 dataset from the National Center for Biotechnology Information (NCBI) GEO database by using an online tool, namely GEO2R (https://www.ncbi.nlm.nih.gov/geo) [27].

\section{Clinical samples}

20 cases of HCC samples including 10 non-cirrhotic HCC and 10 cirrhotic HCC clinical tissues and corresponding normal liver tissues were obtained from 20 patients who had undergo surgery from 2017 to 2018 at the First Affiliated Hospital of Zhejiang University (Hangzhou, China). The study was already obtained the informed consent from each patient and approved by the Ethics Committee of the First Affiliated Hospital of Zhejiang University.

\section{RNA extraction and quantitative polymerase chain reaction (qRT-PCR)}

RNA extraction and qRT-PCR were performed as we previously described [22, 28-30]. Simply, total RNA was extracted from HCC clinical samples by using RNAiso plus Reagent (TaKaRa, Kusatsu, Japan). PrimeScript RT Reagent Kit (TaKaRa, RR0037A) and SYBR Premix Ex Taq (TaKaRa, RR420A) were used to perform qRT-PCR. Gene level was normalized to GAPDH and miRNA level was normalized to U6 gene expression, then relative expression level was analyzed using $2^{-\Delta \Delta C T}$ method. mRNA primers (Additional file 1: Table S1) were purchased from BGI. miRNA reverse transcription primers were synthesized by RiboBio Co. Ltd (Guangzhou, China).

\section{Statistical analysis}

Statistical analysis was conducted by GraphPad prism software (version 7.0.3), and the results were shown as mean \pm SD. Differences between non-cirrhotic HCC and cirrhotic HCC group were analyzed using unpaired Student's $t$-test. Chi square test was employed to assess the relationship between cirrhotic $\mathrm{HCC}$ or non-cirrhotic HCC patients' clinical features and expression of key mRNAs and miRNAs. Only a two-tailed value of $\mathrm{p}<0.05$ was considered as statistically significant.

\section{Results}

Screening and analyzing differentially expressed genes between non-cirrhotic and cirrhotic HCC

A total of 377 HCC patients were found in TCGA database. Among these patients, only 218 cases contain the Ishak score in clinical information. We excluded 159 cases that lack Ishak score information and divided the rest 218 cases into two groups based on their Ishak score. The patients with scores equal or higher than 5 were included in the cirrhotic HCC group $(n=81)$, patients with lower scores than 5 were included in the non-cirrhotic group $(n=137)$. Besides, we found 6 of 377 patients lack information on gene expression, finally we choose 132 noncirrhotic HCC and 79 cirrhotic HCC patients for further analysis, as they contain both enough clinical information and gene expression data. In the next step, based on edge $R$ package analysis and the cut-off criteria (fold Change $\geq 2$, p value $\leq 0.05$ ), 768 differentially expressed genes between non-cirrhotic HCC and cirrhotic HCC were found, including 206 upregulated and 562 downregulated genes (Fig. 1). In addition, clinical characteristics of non-cirrhotic HCC and cirrhotic HCC patients in TCGA were collected, including gender, age at diagnosis, TNM stage, pathologic stage, vascular invasion, HBV infection and HCV infection (Table 1). The analysis showed that the status of gender, age at diagnosis, $\mathrm{T}$ stage, pathologic stage, HBV infection and $\mathrm{HCV}$ infection were obviously different between cirrhotic HCC and non-cirrhotic HCC in TCGA (p<0.05).

\section{GO and KEGG analysis}

To understand the potential biological roles of these differentially expressed genes, three categories of GO functional annotation analysis, containing biological process (BP), cellular component (CC) and molecular function (MF), were analyzed. As shown in Fig. 2a-c, DEGs are significantly enriched in chemical synaptic transmission, epidermis development, anterograde trans-synaptic signaling in the BP category, hormone activity, CXCR chemokine receptor binding, endopeptidase inhibitor activity in the CC category and integral component of plasma membrane, Golgi lumen, azurophil granule lumen in the MF category. KEGG enrichment analysis for these DEGs revealed that neuroactive ligand-receptor interaction, pancreatic secretion and salivary secretion are significantly enriched pathways (Fig. 2d).

(See figure on next page.)

Fig. 1 DEGs between cirrhotic HCC and cirrhotic HCC. a Volcano plot of DEGs between non-cirrhotic HCC and cirrhotic HCC. The red dots represent upregulated genes in cirrhotic HCC $(n=206)$, the green dots represent downregulated genes in cirrhotic HCC $(n=562)$, while the black dots represent genes that are not differentially expressed between two groups. $\mathbf{b}$ The heatmap of differential expressed genes between HCC with or without cirrhosis 


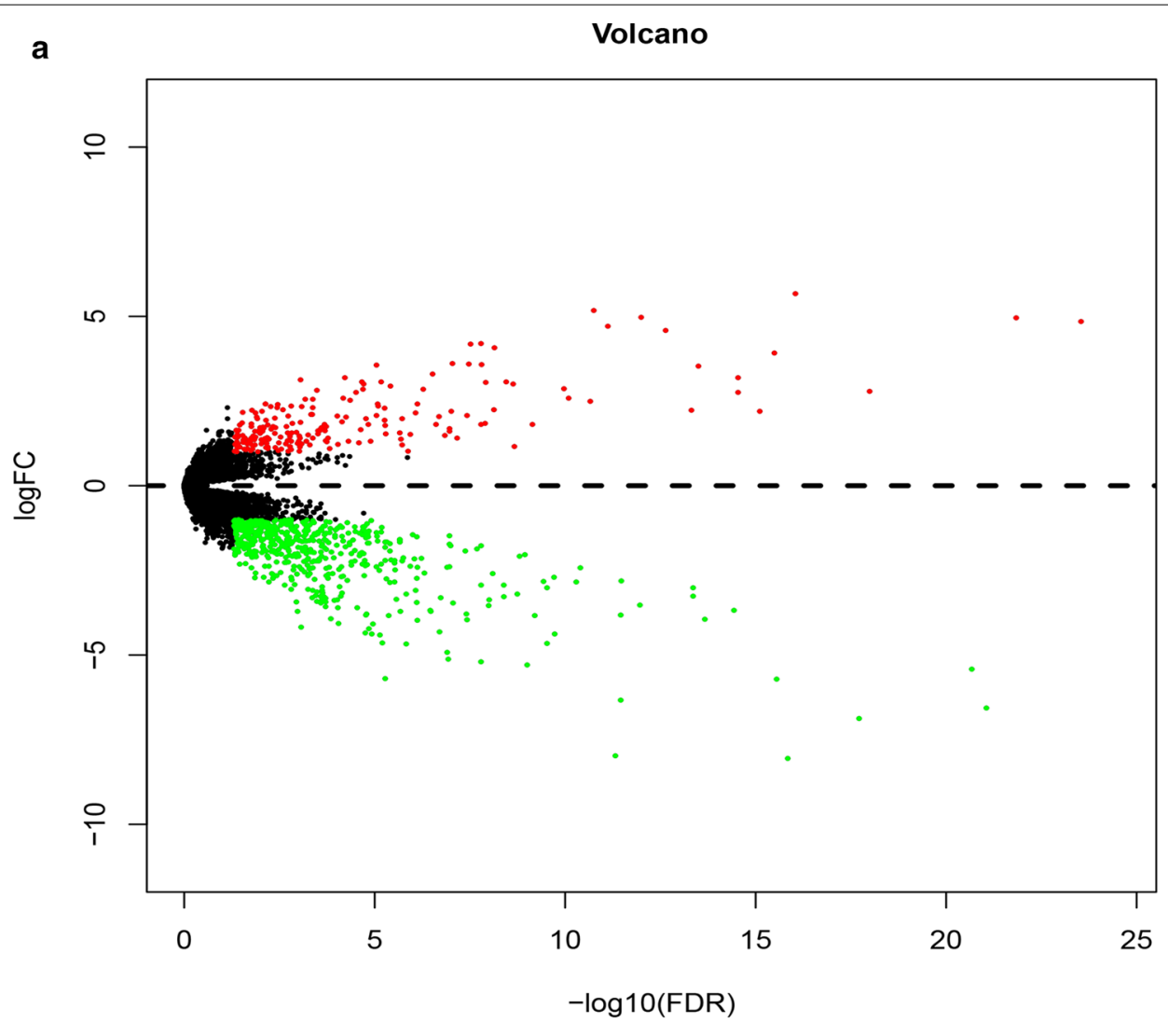

b

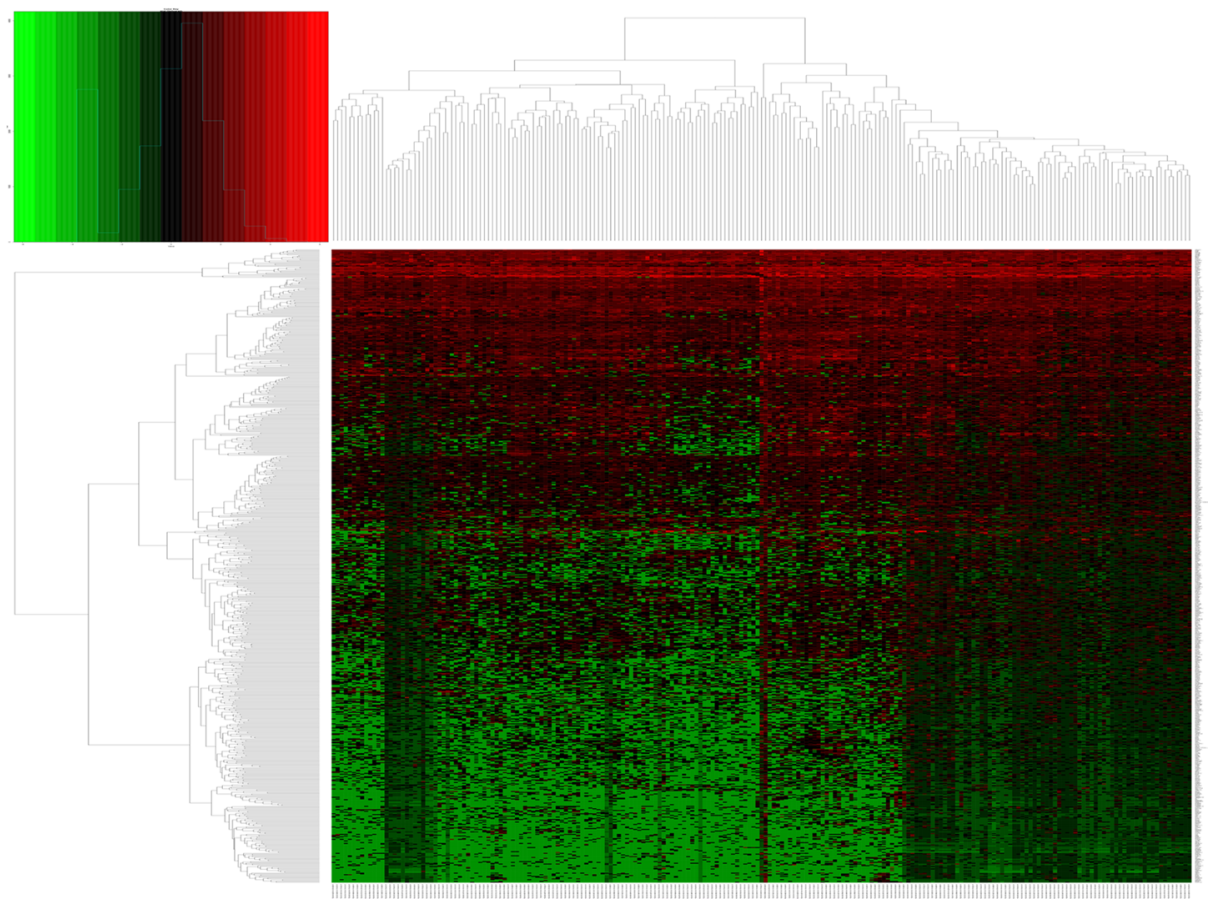


Table 1 Characteristics of non-cirrhotic HCC and cirrhotic HCC patients in TCGA

\begin{tabular}{|c|c|c|c|}
\hline Variables & $\begin{array}{l}\text { Non-cirrhotic HCC } \\
\text { case }(n=132)\end{array}$ & $\begin{array}{l}\text { Cirrhotic HCC } \\
\text { case }(n=79)\end{array}$ & $p$ value \\
\hline \multicolumn{4}{|l|}{ Gender } \\
\hline Male & 82 & 61 & \multirow[t]{2}{*}{0.023} \\
\hline Female & 50 & 18 & \\
\hline \multicolumn{4}{|c|}{ Age at diagnosis } \\
\hline$\geq 60$ & 80 & 36 & \multirow[t]{3}{*}{0.042} \\
\hline$<60$ & 52 & 42 & \\
\hline NA & 0 & 1 & \\
\hline \multicolumn{4}{|l|}{ T stage } \\
\hline $\mathrm{T} 1 / \mathrm{T} 2$ & 97 & 70 & \multirow[t]{4}{*}{0.011} \\
\hline $\mathrm{T} 3 / \mathrm{T} 4$ & 33 & 8 & \\
\hline $\mathrm{TX}$ & 1 & 0 & \\
\hline NA & 1 & 1 & \\
\hline \multicolumn{4}{|l|}{ N stage } \\
\hline NO & 91 & 57 & \multirow[t]{4}{*}{0.681} \\
\hline $\mathrm{N} 1$ & 1 & 1 & \\
\hline NX & 40 & 20 & \\
\hline NA & 0 & 1 & \\
\hline \multicolumn{4}{|l|}{ M stage } \\
\hline Mo & 90 & 62 & \multirow[t]{3}{*}{0.134} \\
\hline M1 & 4 & 0 & \\
\hline$M X$ & 38 & 17 & \\
\hline \multicolumn{4}{|c|}{ Pathologic stage } \\
\hline$|/| \mid$ & 91 & 67 & \multirow[t]{3}{*}{0.013} \\
\hline III/IV & 33 & 9 & \\
\hline NA & 8 & 3 & \\
\hline \multicolumn{4}{|c|}{ Vascular invasion } \\
\hline None & 87 & 52 & \multirow[t]{4}{*}{0.800} \\
\hline Micro & 33 & 22 & \\
\hline Macro & 6 & 2 & \\
\hline NA & 6 & 3 & \\
\hline \multicolumn{4}{|c|}{ HBV infection } \\
\hline Yes & 38 & 36 & \multirow[t]{3}{*}{0.014} \\
\hline No & 88 & 40 & \\
\hline NA & 6 & 3 & \\
\hline \multicolumn{4}{|c|}{ HCV infection } \\
\hline Yes & 14 & 20 & \multirow[t]{3}{*}{0.005} \\
\hline No & 112 & 56 & \\
\hline NA & 6 & 3 & \\
\hline
\end{tabular}

Identify important models and hub genes and validate expression levels, clinical characteristics, prognostic and diagnostic values of the key genes

The DEGs with combined scores higher than 0.4 were selected to construct PPI network using STRING database (Additional file 2: Figure S1). Subsequently, the entire PPI network was analyzed through MCODE, and top three modules were selected for further KEGG pathway enrichment analysis. As shown in Fig. 3, the top three module genes were mostly involved in neuroactive ligand-receptor interaction and mucin type O-Glycan biosynthesis. We set the screening option as Degree Cutoff $=2$, Node Score Cutoff $=0.2$, K-Core $=4$ and Max. Depth $=100$. In the next step, we screened out the top 15 hub nodes ranked by the MCC using CytoHubba plugin, as presented in Fig. 3d. The top 15 hub genes were as follows: LPAR3, CXCL9, CXCL10, CXCL11, PF4, PPBP, CCL19, SST, GAL, NPY1R, CCL25, CCR10, PENK, OPRK1 and CNR1. Additionally, we determined the expression levels of the top 15 hub genes between HCC tissues (non-cirrhotic HCC tissues or cirrhotic HCC tissues) and HCC normal tissues in TCGA. PF4, PPBP were found to be significantly downregulated in cirrhotic HCC than that in paracancerous tissues, while CCL25 was statistically upregulated in non-cirrhotic HCC compared with corresponding normal tissue. Besides, CCL19 was significantly downregulated in non-cirrhotic HCC compared with corresponding normal tissues. Additionally, the expression of CNR1 was higher in both cirrhotic $\mathrm{HCC}$ and non-cirrhotic HCC tissues compared with normal controls (Fig. 4).

In the next step, we validated the prognostic values of 5 key genes in cirrhotic $\mathrm{HCC}$ and non-cirrhotic HCC. We found that all of the non-cirrhotic HCC patients in TCGA contain with completely survival information. However, 1 of 79 cirrhotic HCC patient lack of information on survival, finally we selected 132 non-cirrhotic HCC and 78 cirrhotic HCC patients for survival analysis. Only high CNR1 expression was found to be related to unfavorable overall survival (OS) in cirrhotic HCC (Fig. 4). In addition, a ROC curve (X-axis: 100\%-Specificity\%; Y-axis: Sensitivity\%) was used to estimate the diagnostic values of 5 key genes in $\mathrm{HCC}$ with or without cirrhosis. The results revealed the significant diagnostic values of PF4 and PPBP in cirrhotic HCC, and CCL19, CCL25 in non-cirrhotic HCC, while no significant diagnostic values of CNR1 in $\mathrm{HCC}$ with or without cirrhosis were found (Fig. 4). Finally, Clinical characteristics analysis showed that high expression of CNR1 was greatly correlated with less vascular invasion and fewer HBV infection in non-cirrhotic HCC (Table 2), while high CCL19 expression was positively associated with HBV infection in non-cirrhotic (Table 3). No significant clinical characteristics of PF4, PPBP were found in cirrhotic $\mathrm{HCC}$, neither CCL25 in non-cirrhotic HCC.

\section{Construct differentially miRNA-mRNA networks regulate in HCC with or without cirrhosis}

To find the regulatory mechanisms about non-cirrhotic HCC and cirrhotic HCC, the miRDB database was employed to predict the microRNAs (miRNAs) which 


\section{a}

chemical synaptic transmission (GO:0007268)

epidermis development (GO:0008544)

anterograde trans-synaptic signaling (60:0098916)

ion transport (60:0006811)

transmembrane transport (GO:0055085)

ion transmembrane transport (GO:0034220)

regulation of leukocyte chemotaxis (G0:0002688)

defense response to Gram-negative bacterium (GO:0050829)

sarcomere organization (GO:0045214)

striated muscle contraction (GO:0006941)
C

integral component of plasma membrane (G0:0005887)

\section{Golgi lumen (GO:0005796)}

azurophil granule lumen (GO:0035578)

azurophil granule (GO:0042582)

vacuolar lumen (Go:0005775)

dendrite (GO:0030425)

junctional sarcoplasmic reticulum membrane (G0:0014701)

sarcoplasm (GO:0016528)

primary lysosome (GO:0005766)

intermediate filament (GO:0005882) b

hormone activity (GO:0005179)

\section{CXCR chemokine receptor binding (GO:0045236)}

endopeptidase inhibitor activity (G0:0004866)

peptidase activity, acting on L-amino acid peptides (GO:0070011)

serine-type peptidase activity (GO:0008236)

UDP-galactose:beta-N-acetylglucosamine beta-1,3-galactosyltransferase activity (GO:0008499)

cytokine activity (GO:0005125)

potassium channel activity (GO:0005267)

beta-1,3-galactosyltransferase activity (GO:0048531)

calcium ion binding (GO:0005509) d

Neuroactive ligand-receptor interaction_Homo sapiens_hsa04080

Pancreatic secretion_Homo sapiens_hsa04972

Salivary secretion_Homo sapiens_hsa04970

Protein digestion and absorption_Homo sapien___hsa04974

Gastric acid secretion_Homo sapiens_hsa04971

Glycosphingolipid biosynthesis - lacto and neolacto series_Homo sapiens_hsa00601

Basal cell carcinoma_Homo sapiens_hsa05217

Bile secretion_Homo sapiens_hsa04976

Calcium signaling pathway_Homo sapiens_hsa04020

Cardiac muscle contraction_Homo sapiens_hsa04260

Fig. 2 GO function analysis and KEGG pathway enrichment analysis between non-cirrhotic HCC and cirrhotic HCC. a Biological process (BP) analysis; $\mathbf{b}$ cellular component (CC) analysis; $\mathbf{c}$ molecular function (MF) analysis; $\mathbf{d}$ KEGG pathway enrichment analysis of differential expressed genes between non-cirrhotic HCC and cirrhotic HCC
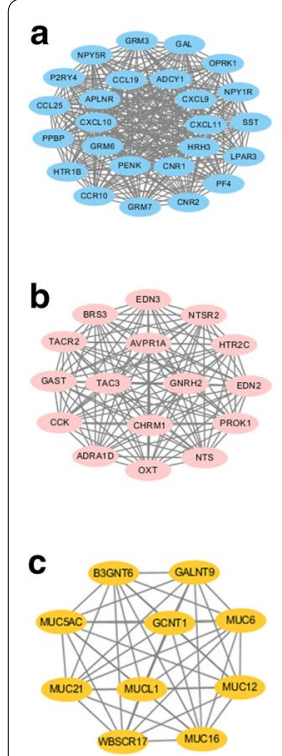
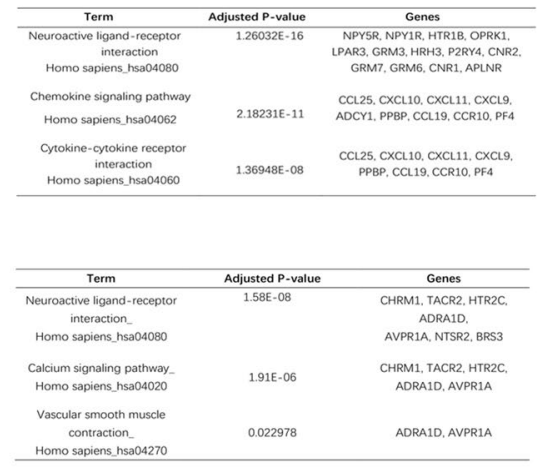

\begin{tabular}{ccc}
\hline Term & Adjusted P-value & Genes \\
\hline $\begin{array}{c}\text { Mucin type O-Glycan } \\
\text { biosynthesis_ }\end{array}$ & $1.97 E-09$ & WBSCR17, B3GNT6, GCNT1, \\
Homo sapiens__sa00512 & & GALNT9 \\
$\begin{array}{c}\text { Metabolic pathways__ } \\
\text { Homo sapiens_hsa01100 }\end{array}$ & 0.002276 & WBSCR17, B3GNT6, GCNT1, \\
GALNT9 \\
\hline
\end{tabular}

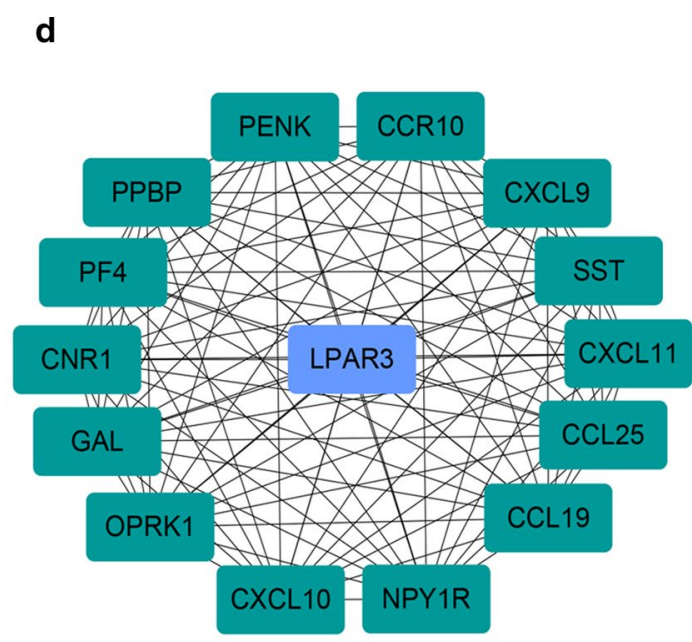

Fig. 3 MCODE analysis, KEGG pathway enrichment analysis and PPI network of the hub genes

\section{(See figure on next page.)}

Fig. 4 Expression analysis, survival analysis and ROC analysis of the 5 key genes between non-cirrhotic HCC and corresponding normal tissues. a CCL19 expression between non-cirrhotic HCC and normal tissues in TCGA; b CCL25 expression between non-cirrhotic HCC and normal tissues in TCGA; c CNR1 expression between non-cirrhotic HCC and normal tissues in TCGA; $\mathbf{d}$ CNR1 expression between cirrhotic HCC and normal tissues in TCGA; e PF4 expression between cirrhotic HCC and normal tissues in TCGA; $\mathbf{f}$ PPBP expression between cirrhotic HCC and normal tissues in TCGA; $\mathbf{g}$ CNR1 survival analysis in cirrhotic HCC; $\mathbf{h}$ CCL19 ROC analysis in non-cirrhotic HCC; $\mathbf{i}$ CCL25 ROC analysis in non-cirrhotic HCC; $\mathbf{g}$ CNR1 ROC analysis in non-cirrhotic HCC; $\mathbf{k}$ CNR1 ROC analysis in cirrhotic HCC; I PF4 ROC analysis in cirrhotic HCC; $\mathbf{m}$ PPBP ROC analysis in cirrhotic HCC 


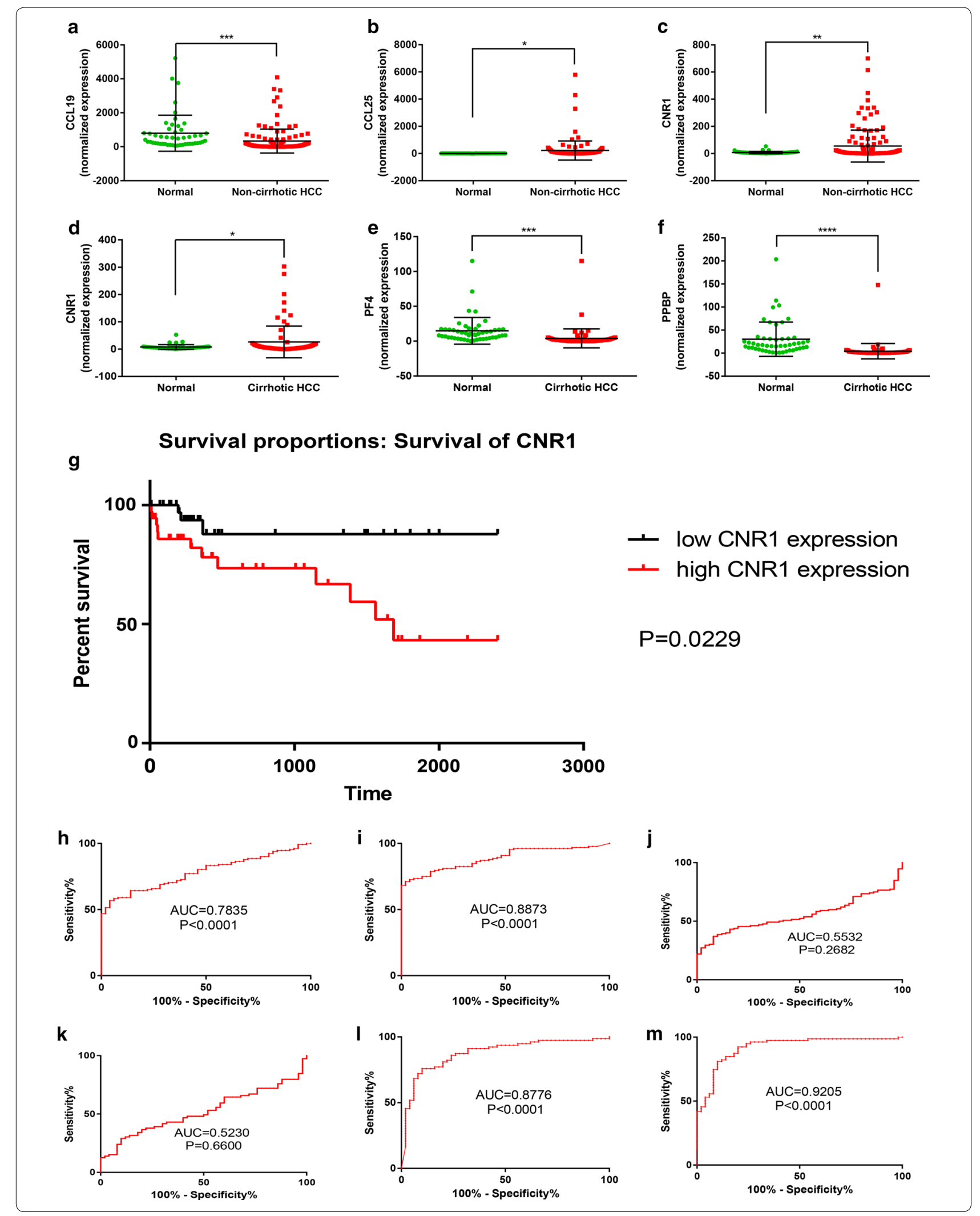


Table 2 Correlations the clinical characteristics among CNR1 between HCC with or without cirrhosis in TCGA

\begin{tabular}{|c|c|c|c|c|c|c|}
\hline \multirow[t]{3}{*}{ Variables } & \multirow[t]{3}{*}{$\mathbf{N}$} & \multirow{2}{*}{\multicolumn{2}{|c|}{$\begin{array}{l}\text { Non-cirrhotic HCC } \\
\text { CNR1 }\end{array}$}} & \multirow[t]{3}{*}{$\mathrm{N}$} & \multicolumn{2}{|l|}{ Cirrhotic HCC } \\
\hline & & & & & \multicolumn{2}{|l|}{ CNR1 } \\
\hline & & $\begin{array}{l}\text { Low/high expression } \\
\text { case }(\mathrm{n})\end{array}$ & $p$ value & & $\begin{array}{l}\text { Low/high expression } \\
\text { case }(\mathrm{n})\end{array}$ & $p$ value \\
\hline \multicolumn{7}{|l|}{ Gender } \\
\hline Male & 82 & $44 / 38$ & 0.282 & 61 & $31 / 30$ & 0.635 \\
\hline Female & 50 & $22 / 28$ & & 18 & $8 / 10$ & \\
\hline \multicolumn{7}{|c|}{ Age at diagnosis } \\
\hline$\geq 60$ & 80 & $38 / 42$ & 0.476 & 36 & $17 / 19$ & 0.650 \\
\hline$<60$ & 52 & $28 / 24$ & & 42 & $22 / 20$ & \\
\hline NA & 0 & 0 & & 1 & $0 / 1$ & \\
\hline \multicolumn{7}{|l|}{ Tstage } \\
\hline $\mathrm{T} 1 / \mathrm{T} 2$ & 97 & $48 / 49$ & 1.000 & 70 & $35 / 35$ & 0.712 \\
\hline $\mathrm{T} 3 / \mathrm{T} 4$ & 33 & $17 / 16$ & & 8 & $3 / 5$ & \\
\hline TX & 1 & $1 / 0$ & & 0 & $0 / 0$ & \\
\hline NA & 1 & $0 / 1$ & & 1 & $1 / 0$ & \\
\hline \multicolumn{7}{|l|}{ N stage } \\
\hline No & 91 & $49 / 42$ & 0.259 & 57 & $29 / 28$ & 1.000 \\
\hline $\mathrm{N} 1$ & 1 & $0 / 1$ & & 1 & $0 / 1$ & \\
\hline NX & 40 & $17 / 23$ & & 20 & $10 / 10$ & \\
\hline NA & 0 & $0 / 0$ & & 1 & $0 / 1$ & \\
\hline \multicolumn{7}{|l|}{ M stage } \\
\hline Mo & 90 & $46 / 44$ & 0.529 & 62 & $31 / 31$ & 0.830 \\
\hline M1 & 4 & $3 / 1$ & & 0 & $0 / 0$ & \\
\hline MX & 38 & $17 / 21$ & & 17 & $8 / 9$ & \\
\hline \multicolumn{7}{|c|}{ Pathologic stage } \\
\hline$|/| \mid$ & 91 & $44 / 47$ & 0.755 & 67 & $35 / 32$ & 0.478 \\
\hline III/IV & 33 & $17 / 16$ & & 9 & $3 / 6$ & \\
\hline NA & 8 & $5 / 3$ & & 3 & $1 / 2$ & \\
\hline \multicolumn{7}{|c|}{ Vascular invasion } \\
\hline None & 87 & $38 / 49$ & 0.127 & 52 & $26 / 26$ & 0.900 \\
\hline Micro & 33 & $18 / 15$ & & 22 & $10 / 12$ & \\
\hline Macro & 6 & $5 / 1$ & & 2 & $1 / 1$ & \\
\hline NA & 6 & $5 / 1$ & & 3 & $2 / 1$ & \\
\hline \multicolumn{7}{|c|}{ HBV infection } \\
\hline Yes & 38 & $25 / 13$ & 0.036 & 36 & $20 / 16$ & 0.358 \\
\hline No & 88 & $40 / 48$ & & 40 & $18 / 22$ & \\
\hline NA & 6 & $1 / 5$ & & 3 & $1 / 2$ & \\
\hline \multicolumn{7}{|c|}{ HCV infection } \\
\hline Yes & 14 & $6 / 8$ & 0.488 & 20 & $13 / 7$ & 0.118 \\
\hline No & 112 & $59 / 53$ & & 56 & $25 / 31$ & \\
\hline NA & 6 & $1 / 5$ & & 3 & $1 / 2$ & \\
\hline
\end{tabular}

NA not applicable

The significant $p$ value is marked with italic type 
Table 3 Correlations the clinical characteristics among CCL19 and non-cirrhotic HCC in TCGA

\begin{tabular}{|c|c|c|c|}
\hline \multirow[t]{3}{*}{ Variables } & \multirow[t]{3}{*}{$\mathbf{N}$} & \multicolumn{2}{|l|}{ Non-cirrhotic HCC } \\
\hline & & \multicolumn{2}{|l|}{ CCL19 } \\
\hline & & $\begin{array}{l}\text { Low/high expression } \\
\text { case }(\mathrm{n})\end{array}$ & $\mathrm{p}$ value \\
\hline \multicolumn{4}{|l|}{ Gender } \\
\hline Male & 82 & $64 / 18$ & \multirow[t]{2}{*}{0.585} \\
\hline Female & 50 & $41 / 9$ & \\
\hline \multicolumn{4}{|c|}{ Age at diagnosis } \\
\hline$\geq 60$ & 80 & $68 / 12$ & \multirow[t]{2}{*}{0.054} \\
\hline$<60$ & 52 & $37 / 15$ & \\
\hline \multicolumn{4}{|l|}{ T stage } \\
\hline $\mathrm{T} 1 / \mathrm{T} 2$ & 97 & $76 / 21$ & \multirow[t]{4}{*}{0.846} \\
\hline $\mathrm{T} 3 / \mathrm{T} 4$ & 33 & $27 / 6$ & \\
\hline TX & 1 & $1 / 0$ & \\
\hline NA & 1 & $1 / 0$ & \\
\hline \multicolumn{4}{|l|}{ N stage } \\
\hline No & 91 & $73 / 18$ & \multirow[t]{3}{*}{0.853} \\
\hline $\mathrm{N} 1$ & 1 & $1 / 0$ & \\
\hline NX & 40 & $31 / 9$ & \\
\hline \multicolumn{4}{|l|}{ M stage } \\
\hline MO & 90 & $74 / 16$ & \multirow[t]{3}{*}{0.178} \\
\hline M1 & 4 & $2 / 2$ & \\
\hline$M X$ & 38 & $29 / 9$ & \\
\hline \multicolumn{4}{|c|}{ Pathologic stage } \\
\hline$|/| \mid$ & 91 & $72 / 19$ & \multirow[t]{3}{*}{0.741} \\
\hline III/IV & 33 & $27 / 6$ & \\
\hline NA & 8 & $6 / 2$ & \\
\hline \multicolumn{4}{|c|}{ Vascular invasion } \\
\hline None & 87 & $69 / 18$ & \multirow[t]{4}{*}{1.000} \\
\hline Micro & 33 & $26 / 7$ & \\
\hline Macro & 6 & $5 / 1$ & \\
\hline NA & 6 & $5 / 1$ & \\
\hline \multicolumn{4}{|c|}{ HBV infection } \\
\hline Yes & 38 & $27 / 11$ & \multirow[t]{3}{*}{0.176} \\
\hline No & 88 & $72 / 16$ & \\
\hline NA & 6 & $6 / 0$ & \\
\hline \multicolumn{4}{|c|}{ HCV infection } \\
\hline Yes & 14 & $7 / 7$ & \multirow[t]{3}{*}{0.016} \\
\hline No & 112 & $92 / 20$ & \\
\hline NA & 6 & $6 / 0$ & \\
\hline
\end{tabular}

NA not applicable

The significant $p$ value is marked with italic type

could regulate 5 target genes. Besides, we intended to further evaluate the correlation between predicted miRNAs and corresponding target genes using the starBase database. As we all know, target genes were conversely regulated by miRNAs, and the higher correlation coefficient (r), the greater mutual regulation effect. Thus, we chose those predicted miRNAs that negatively correlated with target genes and the $|\mathrm{r}|>0.1$, $\mathrm{p}$-value $<0.05$ for the next research. The analytic results showed in Fig. 5 indicated that CCL19 could be potentially modulated by miR-30c-5p, miR-30e-5p and miR-6509-5p. miR-30a-5p and miR-1287-5p could potentially target CCL25. In addition, miR-3662, miR-4795-3p and miR6783-3p could potentially target PPBP. No predicted miRNAs of PF4 met the conditions. As for CNR1, $7 \mathrm{miR}$ NAs including miR-4482-3p, miR-221-3p, miR-30d-5p, miR-194-5p, miR-98-3p, let-7b-3p and let-7f-1-3p were selected as potential negative regulators. After that, we analyzed those miRNAs expression between corresponding normal liver tissues and HCC tissues with or without cirrhosis in GSE10694 database from GEO, which was included 40 cirrhotic HCC patients and 38 non-cirrhotic HCC patients [31]. Based on this analysis, we found that miR-194-5p was downregulated in HCC tissues with or without cirrhosis than corresponding normal liver tissues. The expression level of miR-30c-5p was downregulated in non-cirrhotic $\mathrm{HCC}$ patients compared with corresponding non-cirrhotic normal liver tissues, while miR-30e-5p and miR-30a-5p were also downregulated in non-cirrhotic HCC tissues compared with corresponding non-cirrhotic normal liver tissues, not as expected (not list Table 4). Besides, let-7b-3p and let-7f-1-3p expression were higher in cirrhotic normal liver tissues than in cirrhotic HCC tissues (Table 4). Finally, combined with the expression levels of the key miRNAs between HCC with or without cirrhosis and normal tissues, the key miRNAmRNA regulatory networks in non-cirrhotic HCC and cirrhotic HCC were constructed (Fig. 6).

To validate the values of these key miRNAs and target genes, we further detected the expression levels in 20 pairs of HCC clinical samples (10 cirrhotic HCC and 10 non-cirrhotic HCC) and compared with corresponding normal liver tissues. As the results shown in Fig. 7, qRT-PCR indicated that PF4 and PPBP were downregulated in cirrhotic HCC tissues compared with normal liver tissues. CNR1 was upregulated in both cirrhotic HCC and non-cirrhotic HCC tissues. Besides, CCL19 was downregulated in non-cirrhotic HCC, while no significant value of CCL25 was found between non-cirrhotic HCC and corresponding normal liver samples. In addition, miR-194-5p and let-7f-1-3p were downregulated in $\mathrm{HCC}$ with or without cirrhosis than in paracancerous tissues. What's more, let-7b-3p was downregulated in cirrhotic HCC compared to matched normal liver tissues, while not in non-cirrhotic HCC. Additionally, compared with normal liver tissues, the expression levels of miR30a-5p was significantly decreased in non-cirrhotic HCC. All these findings suggested that miR-194-5p/CNR1 and 
a

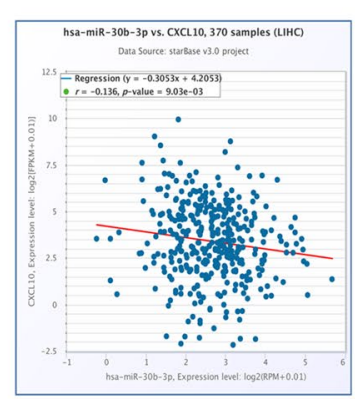

e

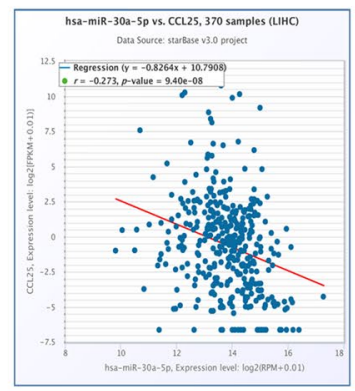

i

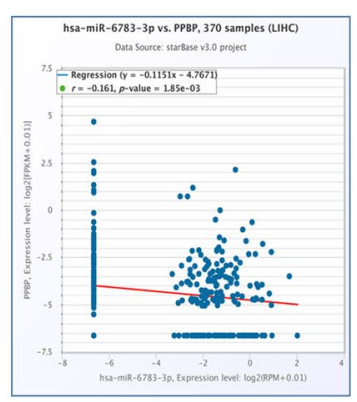

b

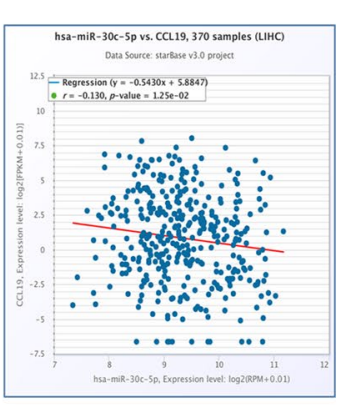

\section{$f$}

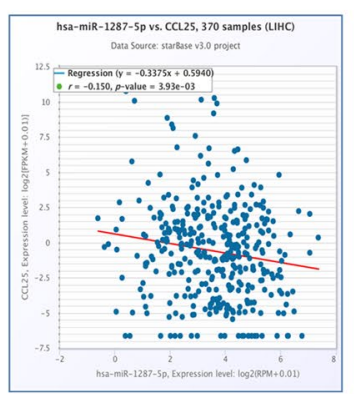

j

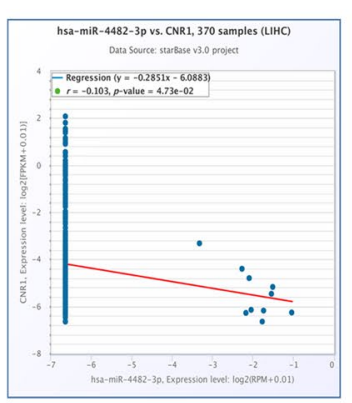

c

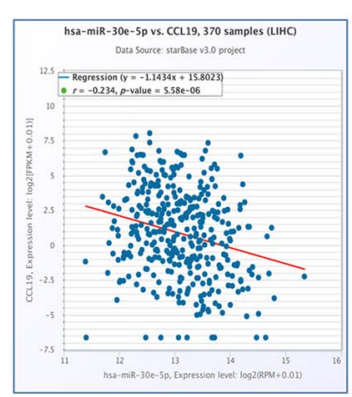

9

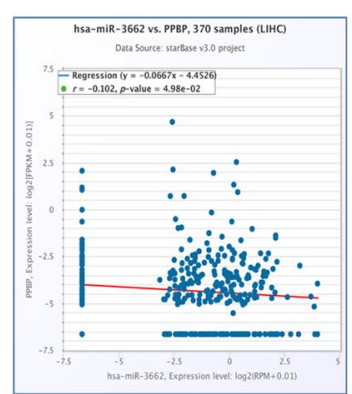

k

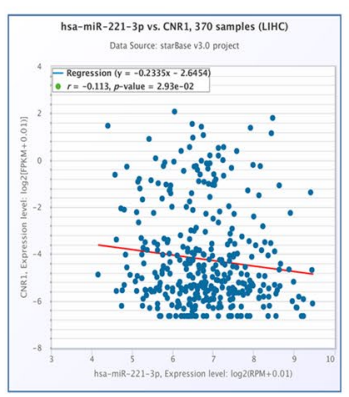

d

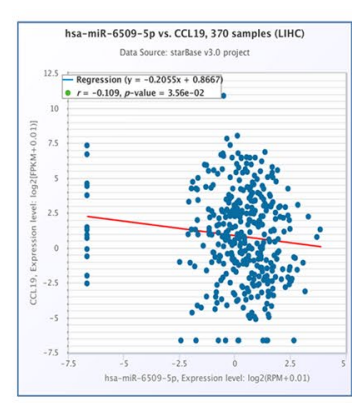

h
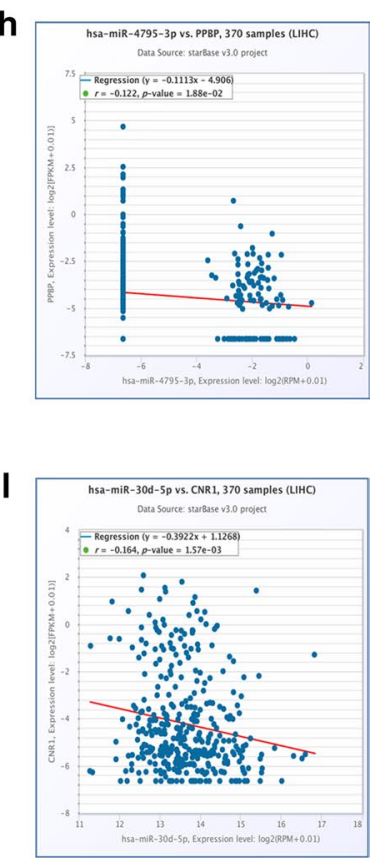

m

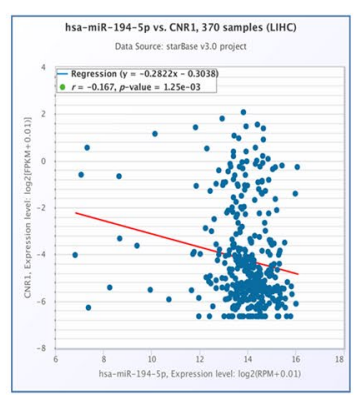

n

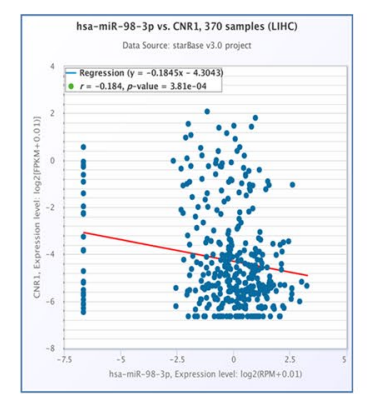

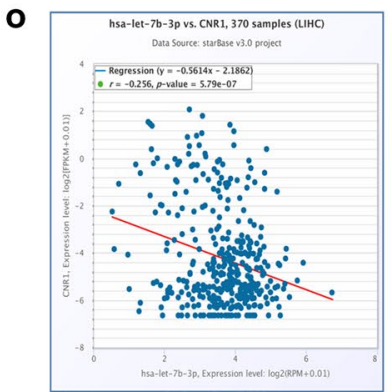

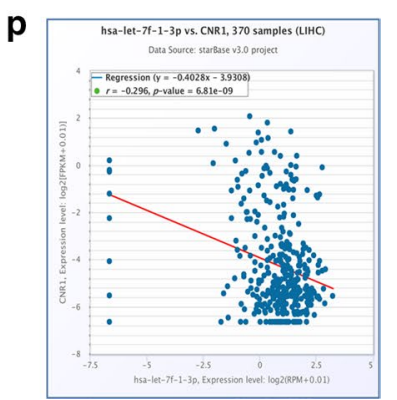

Fig. 5 Correlation analysis between 517 candidate target miRNAs from miRDB database and 7 key DEGs by using starBase database (only 16 significant correlation analysis were showed). a hsa-miR-30a-5p vs. CCL25; b hsa-miR-1287-5p vs. CCL25; c hsa-miR-3662 vs. PPBP; d hsa-miR-4795-3p vs. PPBP; e hsa-miR-6783-3p vs. PPBP; f hsa-miR-4482-3p vs. CNR1; $\mathbf{g}$ hsa-miR-221-3p vs. CNR1; $\mathbf{h}$ hsa-miR-30d-5p vs. CNR1; i hsa-miR-194-5p vs. CNR1; $\mathbf{g}$ hsa-miR-98-3p vs. CNR1; $\mathbf{k}$ hsa-let-7b-3p vs. CNR1; I hsa-let-7f-1-3p vs. CNR1; $\mathbf{m}$ hsa-miR-30c-5p vs. CCL19; $\mathbf{n}$ hsa-miR-30e-5p vs. CCL19; $\mathbf{0}$ hsa-miR-6509-5p vs. CCL19 


Table 4 Key miRNAs significantly differentially
expressed in cirrhotic HCC or cirrhotic HCC compared
with corresponding normal liver tissues in GSE10694
from GEO database

\begin{tabular}{lllll}
\hline GSE10694 & adj.p.Val & logFC & miRNA_ID & Target gene \\
\hline $\begin{array}{c}\text { Non-cirrhotic HCC } \\
\text { Downregulated }\end{array}$ & $3.43 E-01$ & 0.2545 & hsa-miR-194-5p & CNR1 \\
Downregulated & $9.32 E-02$ & 0.29473 & hsa-miR-30c-3p & CCL19 \\
Cirrhotic HCC & & & & \\
Downregulated & $5.72 E-01$ & 0.20674 & hsa-miR-194-5p & CNR1 \\
Downregulated & $6.73 E-01$ & 0.17521 & hsa-let-7f-1-3p & CNR1 \\
Downregulated & $6.90 E-01$ & 0.15632 & hsa-let-7b-3p & CNR1 \\
\hline
\end{tabular}

NA not applicable

let-7f-1-3p/CNR1 axes may play important roles in both non-cirrhotic HCC and cirrhotic HCC. Let-7b-3p/CNR1 axis may exert biological effect in cirrhotic HCC. Furthermore, CCL19 and miR-30a-5p were also worth to research in non-cirrhotic HCC, as well as PPBP and PF4 in cirrhotic HCC.

\section{Discussion}

As the most common malignancy in the liver, hepatocellular carcinoma includes two kinds of types, non-cirrhotic HCC and cirrhotic HCC. HCC patients with or without cirrhosis present different clinical characteristics, and regulated by various miRNA-mRNA networks. However, few researches directly investigated the underlying molecular mechanisms between cirrhotic HCC and non-cirrhotic HCC. Therefore, it's meaningful to explore the differences between two kinds of types in HCC.

In this study, we discovered DEGs between cirrhotic $\mathrm{HCC}$ and non-cirrhotic HCC by performing a differential expression analysis based on the data from TCGA. Next, by comparing 15 hub genes expression levels in the cirrhotic HCC or non-cirrhotic HCC tissues with corresponding normal tissues expression levels in TCGA, we found 5 key DEGs between two kinds of types in HCC. Furthermore, after validating the expression of predicted miRNAs between HCC (cirrhosis or non-cirrhosis) and corresponding normal liver tissues (cirrhosis or non-cirrhosis) in GEO database, we selected key predicted miRNAs for 5 key genes and first constructed the miRNA-mRNA regulatory networks in HCC with or without cirrhosis. In the next step, we further analyzed the expression levels of these key miRNAs and genes in clinical samples.

Our study first reported that high expression of CNR1 was associated with worth OS in cirrhotic HCC but not in non-cirrhotic HCC, and correlated with less vascular invasion and fewer HBV infection in non-cirrhotic HCC. Besides, CNR1 expression was upregulated in HCC with or without cirrhosis than that in compared normal tissues both in TCGA and qRT-PCR results. What's more, Liu et al. [32] demonstrated that loss of cannabinoid receptor 1 (CNR1) expression could reduce liver-specific gene expression, thus leading to smaller livers. Additionally, our analytic results showed that CNR1 was an independent risk indicator for the prognosis of cirrhotic HCC patients but not of non-cirrhotic HCC patients,

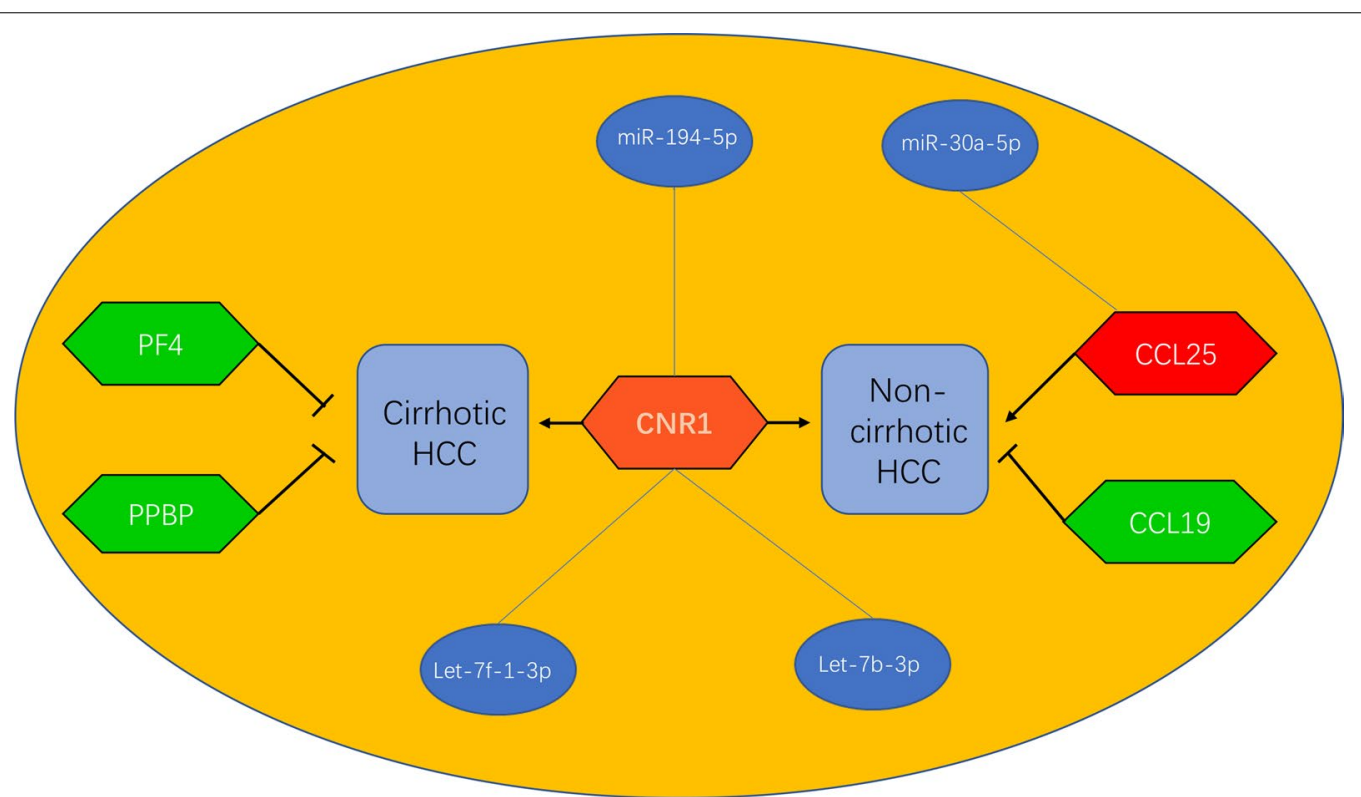

Fig. 6 miRNA-mRNA regulate networks constructed in non-cirrhotic HCC and cirrhotic HCC 

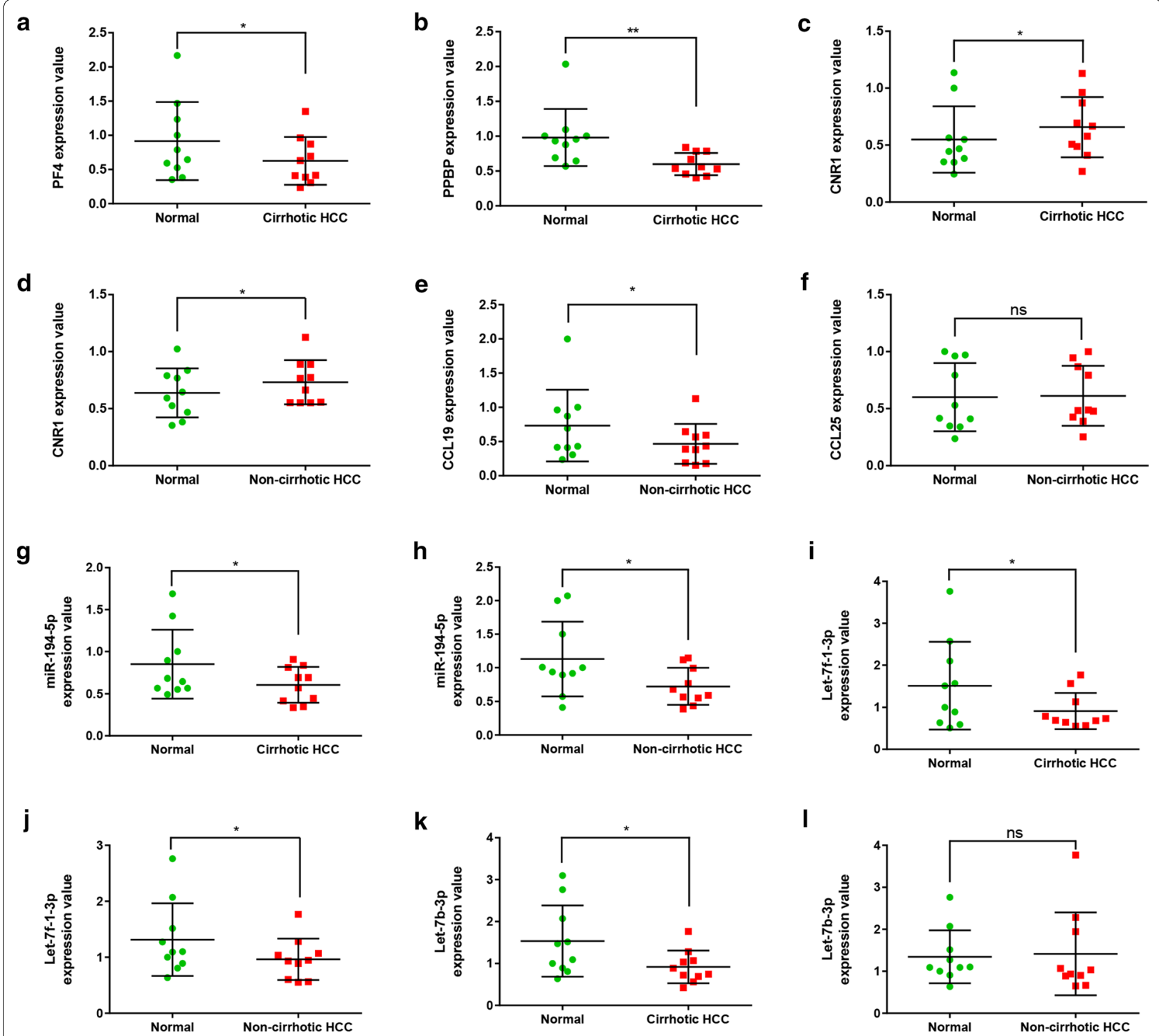

m

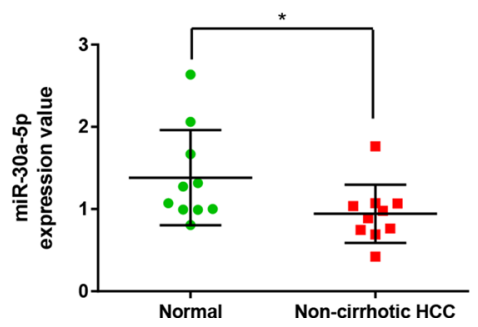

Fig. 7 The expression levels of potential miRNAs and target genes in cirrhotic HCC or non-cirrhotic HCC tissues compared to matched normal tissues. a PF4 expression between cirrhotic HCC and normal tissues; $\mathbf{b}$ PPBP expression between cirrhotic HCC and normal tissues; $\mathbf{c}$ CNR1 expression between cirrhotic HCC and normal tissues; $\mathbf{d}$ CNR1 expression between non-cirrhotic HCC and normal tissues; e CCL19 expression between non-cirrhotic HCC and normal tissues; $\mathbf{f} C C L 25$ expression between non-cirrhotic HCC and normal tissues; $\mathbf{g}$ miR-194-5p expression between cirrhotic HCC and normal tissues; $\mathbf{h}$ miR-194-5p expression between non-cirrhotic HCC and normal tissues; $\mathbf{i}$ let-7f-1-3p expression between cirrhotic HCC and normal tissues; $\mathbf{g}$ let-7f-1-3p expression between non-cirrhotic HCC and normal tissues; $\mathbf{k}$ let-7b-3p expression between cirrhotic HCC and normal tissues; I let-7b-3p expression between non-cirrhotic HCC and normal tissues; $\mathbf{m}$ miR-30a-5p expression between non-cirrhotic HCC and normal tissues 
indicating that two kinds of HCC types may involve separate risk factors. Meanwhile, CNR1 might be an important tumor promoter in HCC. It's unexpected that CNR1 expression was higher in non-cirrhotic HCC tissues than corresponding normal tissues in TCGA, whereas associated with less vascular invasion and fewer HBV infection in non-cirrhotic HCC. Thus, additional studies of CNR1 are necessary to investigate the expression and function in HCC including cirrhotic HCC and non-cirrhotic HCC in the future.

What's more, ROC analysis revealed that CCL19, CCL25 may play important roles in non-cirrhotic HCC diagnosis and PF4, PPBP have important diagnostic values in cirrhotic HCC, but no diagnostic values of CNR1 in cirrhotic HCC and non-cirrhotic HCC were found. Clinical characteristics analysis revealed that higher expression level of CCL19 was associated with higher HCV infection. QRT-PCR results found important values of CCL19 in non-cirrhotic HCC, PF4 and PPBP in cirrhotic HCC, while without CCL25. All of the results indicated that these genes play important roles in HCC diagnosis, and worth to research in $\mathrm{HCC}$ with or without cirrhosis. It's also surprising to find that let-7f-1-3p and let-7b-3p which were predicted to target $C N R 1$ have no significant differentially expressed values between noncirrhotic $\mathrm{HCC}$ tissues and corresponding normal liver tissues in GEO database. Because of limited clinical data, more studies are needed to investigate this.

After reviewing previous articles, we found that lots of researches in HCC have already been launched to investigate the roles of the mRNAs and miRNAs involved in the constructed networks. For example, CCL25 could promote invasion and migration of HCC [33]. Qin et al. [34] indicated that miR-30e-5p could inhibit HBV replication. Besides, let-7b-3p and miR-30c-5p may be useful biomarkers of early hepatitis B virus-related HCC and HCVrelated HCC patients, respectively $[35,36]$. Whereas, CCL19 inhibited the proliferation and migration ability of HCC cells [37]. miR-194-5p accelerated the progression of HBV-related liver disease [38]. Although PF4 does not seem to be a prognostic or diagnostic biomarker in HCC for cirrhotic patients [39], our research found that PF4 may play an important role in cirrhotic-HCC. However, the functions of PPBP, CCL25, miR-30a-5p and let-7f$1-3 p$ in $\mathrm{HCC}$ are still not clearly. Furthermore, researches about the mRNAs and miRNAs involved in constructed networks aiming at specific types of HCC (cirrhotic HCC or non-cirrhotic HCC) are still extremely limited.

It's obvious that differential miRNA-mRNA regulatory networks are greatly participated in HCC progression including cirrhotic HCC and non-cirrhotic HCC. Those key genes and miRNAs in our study are closely related with prognostic values, diagnostic values and some specific clinical characteristics in HCC patients, which can make us better understand the mechanisms between non-cirrhotic $\mathrm{HCC}$ and cirrhotic $\mathrm{HCC}$ and provide effective and promising approaches in treating HCC. However, there are still some limitations in this study, such as (1) only top 15 hub genes was validated for the further research; (2) lack of research on detailed molecular mechanisms that the key mRNAs and key miRNAs regulate in HCC with or without cirrhosis; (3) some function studies about mRNAs and miRNAs in the constructed networks were not as excepted, and lots of mRNAs and miRNAs were lack of research. Even so, our finding remains useful, as the differential miRNA-mRNA regulatory networks in HCC patients with or without cirrhosis may provide more helpful biomarkers, improve prognosis and therapy of HCC patients in further.

\section{Conclusion}

In conclusion, we first successfully constructed the miRNA-mRNA networks between cirrhotic HCC and non-cirrhotic HCC by using bioinformatic analysis and preliminary experimental validation. The present study suggested that CNR1, negatively regulated by miR194-5p and let-7f-1-3p, might be a promising factor of cirrhotic and non-cirrhotic HCC patients. Let-7b-3p may regulate CNR1 participant in cirrhotic $\mathrm{HCC}$ progression. miR-30a-5p-CCL25 axis may play an important role in promoting non-cirrhotic HCC progression. In addition, PF4 and PPBP may function as tumor suppressors in cirrhotic HCC, meanwhile, CCL19 may act as a protective factor in non-cirrhotic $\mathrm{HCC}$, while miRNAs regulate mechanisms still unclear, further experimental trials are still need to be launched in the future.

\section{Additional files}

Additional file 1: Table S1. Primers of key mRNAs.

Additional file 2: Figure S1. PPI network of DEGs between cirrhotic HCC and non-cirrhotic HCC by using STRING database.

\section{Acknowledgements}

Not applicable.

\section{Authors' contributions}

BD, WL and JL: main text and illustration; RL: language; JC and WF: concept, discussion. All authors read and approved the final manuscript.

\section{Funding}

This research was supported by the National Natural Science Foundation of China $(81572987,81874225)$ and Department of Science and Technology of Zhejiang Province (Grant No. 2014C03012). 


\section{Availability of data and materials \\ Not applicable.}

\section{Ethics approval and consent to participate}

Not applicable.

\section{Patient consent for publication}

Not applicable.

\section{Competing interests}

The authors declare that they have no competing interests.

\section{Author details}

${ }^{1}$ Program of Innovative Cancer Therapeutics, Division of Hepatobiliary and Pancreatic Surgery, Department of Surgery, First Affiliated Hospital, College of Medicine, Key Laboratory of Combined Multi-Organ Transplantation, Ministry of Public Health, Key Laboratory of Organ Transplantation, Zhejiang University, Hangzhou 310003, Zhejiang Province, China. ${ }^{2}$ Department of Intensive Care Unit, Changxing People's Hospital of Zhejiang Province, Huzhou 313100, Zhejiang, China. ${ }^{3}$ Department of Critical Care Medicine, The Second Affiliated Hospital of Xi'an Jiaotong University, Xi'an 710004, Shanxi, China. ${ }^{4}$ First Affiliated Hospital of Jiaxing University, Jiaxing 314000, Zhejiang, China.

Received: 23 February 2019 Accepted: 10 July 2019

Published online: 18 July 2019

\section{References}

1. Forner A, Reig M, Bruix J. Hepatocellular carcinoma. Lancet (London, England). 2018;391(10127):1301-14.

2. Torre LA, Bray F, Siegel RL, Ferlay J, Lortet-Tieulent J, Jemal A. Global cancer statistics, 2012. CA Cancer J Clin. 2015;65(2):87-108.

3. Fujiwara N, Friedman SL, Goossens N, Hoshida Y. Risk factors and prevention of hepatocellular carcinoma in the era of precision medicine. J Hepatol. 2018;68(3):526-49.

4. Okuda K, Nakashima T, Sakamoto K, Ikari T, Hidaka H, Kubo Y, Sakuma K, Motoike Y, Okuda H, Obata H. Hepatocellular carcinoma arising in noncirrhotic and highly cirrhotic livers: a comparative study of histopathology and frequency of hepatitis B markers. Cancer. 1982;49(3):450-5.

5. Lin CW, Lin CC, Mo LR, Chang CY, Perng DS, Hsu CC, Lo GH, Chen YS, Yen $Y C, H u$ JT, et al. Heavy alcohol consumption increases the incidence of hepatocellular carcinoma in hepatitis B virus-related cirrhosis. J Hepatol. 2013;58(4):730-5.

6. Karageorgos SA, Stratakou S, Koulentaki M, Voumvouraki A, Mantaka A, Samonakis D, Notas G, Kouroumalis EA. Long-term change in incidence and risk factors of cirrhosis and hepatocellular carcinoma in Crete, Greece: a 25-year study. Ann Gastroenterol. 2017;30(3):357-63.

7. Lee NCW, Carella MA, Papa S, Bubici C. High expression of glycolytic genes in cirrhosis correlates with the risk of developing liver cancer. Front Cell Dev Biol. 2018;6:138.

8. Fattovich G, Stroffolini T, Zagni I, Donato F. Hepatocellular carcinoma in cirrhosis: incidence and risk factors. Gastroenterology. 2004;127(5 Suppl 1):S35-50.

9. Mohamad B, Shah V, Onyshchenko M, Elshamy M, Aucejo F, Lopez R, Hanouneh IA, Alhaddad R, Alkhouri N. Characterization of hepatocellular carcinoma (HCC) in non-alcoholic fatty liver disease (NAFLD) patients without cirrhosis. Hepatol Int. 2016;10(4):632-9.

10. Desai A, Sandhu S, Lai JP, Sandhu DS. Hepatocellular carcinoma in noncirrhotic liver: a comprehensive review. World J Hepatol. 2019;11(1):1-18.

11. Kumar M, Kumar R, Hissar SS, Saraswat MK, Sharma BC, Sakhuja P, Sarin SK. Risk factors analysis for hepatocellular carcinoma in patients with and without cirrhosis: a case-control study of 213 hepatocellular carcinoma patients from India. J Gastroenterol Hepatol. 2007;22(7):1104-11.

12. Kozbial K, Moser S, Schwarzer R, Laferl H, Al-Zoairy R, Stauber R, Stattermayer AF, Beinhardt S, Graziadei I, Freissmuth C, et al. Unexpected high incidence of hepatocellular carcinoma in cirrhotic patients with sustained virologic response following interferon-free direct-acting antiviral treatment. J Hepatol. 2016;65(4):856-8.
13. Nguyen $M H_{\text {, }}$ Yang HI, Le A, Henry L, Nguyen $\mathrm{N}$, Lee MH, Zhang J, Wong C, Wong C, Trinh H. Reduced incidence of hepatocellular carcinoma with tenofovir in chronic hepatitis B patients with and without cirrhosis - a propensity score matched study. J Infect Dis. 2018;219(1):10-8.

14. Trinchet JC. Surveillance for hepatocellular carcinoma in cirrhotic patients: from official recommendations to the real life. J Hepatol. 2011:54(6):1310-1.

15. Pang Q, Zhang JY, Xu XS, Song SD, Chen W, Zhou YY, Miao RC, Qu K, Liu SS, Dong YF, et al. The prognostic values of 12 cirrhosis-relative noninvasive models in patients with hepatocellular carcinoma. Scand J Clin Lab Invest. 2015;75(1):73-84.

16. Guo H, Ingolia NT, Weissman JS, Bartel DP. Mammalian microRNAs predominantly act to decrease target mRNA levels. Nature. 2010;466(7308):835-40.

17. Huntzinger E, Izaurralde E. Gene silencing by microRNAs: contributions of translational repression and mRNA decay. Nat Rev Genet. 2011;12(2):99-110.

18. Guo L, Zhao Y, Yang S, Zhang H, Chen F. Integrative analysis of miRNA-mRNA and miRNA-miRNA interactions. Biomed Res Int. 2014:2014:907420.

19. Bajan S, Hutvagner G. Regulation of miRNA processing and miRNA mediated gene repression in cancer. MicroRNA (Shariqah, United Arab Emirates). 2014;3(1):10-7.

20. Goodman ZD. Grading and staging systems for inflammation and fibrosis in chronic liver diseases. J Hepatol. 2007:47(4):598-607.

21. Lou W, Ding B, Xu L, Fan W. Construction of potential glioblastoma multiforme-related miRNA-mRNA regulatory network. Front Mol Neurosci. $2019 ; 12: 66$

22. Lou W, Liu J, Ding B, Jin L, Xu L, Li X, Chen J, Fan W. Five miRNAs-mediated PIEZO2 downregulation, accompanied with activation of Hedgehog signaling pathway, predicts poor prognosis of breast cancer. Aging. 2019;11(9):2628-52.

23. Szklarczyk D, Morris JH, Cook H, Kuhn M, Wyder S, Simonovic M, Santos A, Doncheva NT, Roth A, Bork P, et al. The STRING database in 2017: quality-controlled protein-protein association networks, made broadly accessible. Nucleic Acids Res. 2017;45(D1):D362-D368.

24. Fan $Y$, Xia J. miRNet-functional analysis and visual exploration of miRNAtarget interactions in a network context. Methods Mol Biol (Clifton, NJ). 2018;1819:215-33.

25. Wang X. Improving microRNA target prediction by modeling with unambiguously identified microRNA-target pairs from CLIP-ligation studies. Bioinformatics (Oxford, England). 2016:32(9):1316-22.

26. Zhou H, Li J-H, Liu S, Qu L-H, Yang J-H. starBase v2.0: decoding miRNAceRNA, miRNA-ncRNA and protein-RNA interaction networks from largescale CLIP-Seq data. Nucleic Acids Res. 2013;42(D1):D92-7.

27. Edgar R, Barrett T. NCBI GEO standards and services for microarray data. Nat Biotechnol. 2006;24(12):1471-2.

28. Lou W, Chen J, Ding B, Chen D, Zheng H, Jiang D, Xu L, Bao C, Cao G, Fan W. Identification of invasion-metastasis-associated microRNAs in hepatocellular carcinoma based on bioinformatic analysis and experimental validation. J Transl Med. 2018;16(1):266.

29. Lou W, Liu J, Ding B, Chen D, Xu L, Ding J, Jiang D, Zhou L, Zheng S, Fan W. Identification of potential miRNA-mRNA regulatory network contributing to pathogenesis of HBV-related HCC. J Transl Med. 2019;17(1):7.

30. Lou W, Liu J, Ding B, Xu L, Fan W. Identification of chemoresistance-associated miRNAs in breast cancer. Cancer Manag Res. 2018;10:4747-57.

31. Li W, Xie L, He X, Li J, Tu K, Wei L, Wu J, Guo Y, Ma X, Zhang P, et al. Diagnostic and prognostic implications of microRNAs in human hepatocellular carcinoma. Int J Cancer. 2008;123(7):1616-22.

32. Liu LY, Alexa K, Cortes M, Schatzman-Bone S, Kim AJ, Mukhopadhyay B, Cinar R, Kunos G, North TE, Goessling W. Cannabinoid receptor signaling regulates liver development and metabolism. Development (Cambridge, England). 2016;143(4):609-22.

33. Zhang Z, Sun T, Chen Y, Gong S, Sun X, Zou F, Peng R. CCL $25 / C C R 33$ signal promotes migration and invasion in hepatocellular and breast cancer cell lines. DNA Cell Biol. 2016:35(7):348-57.

34. Qin X, Li C, Guo T, Chen J, Wang HT, Wang YT, Xiao YS, Li J, Liu P, Liu ZS, et al. Upregulation of DARS2 by HBV promotes hepatocarcinogenesis through the miR-30e-5p/MAPK/NFAT5 pathway. J Exp Clin Cancer Res. 2017:36(1):148 
35. Oksuz Z, Serin MS, Kaplan E, Dogen A, Tezcan S, Aslan G, Emekdas G, Sezgin O, Altintas E, Tiftik EN. Serum microRNAs; miR-30c-5p, miR-223-3p, miR-302c-3p and miR-17-5p could be used as novel non-invasive biomarkers for HCV-positive cirrhosis and hepatocellular carcinoma. Mol Biol Rep. 2015;42(3):713-20.

36. Hung $\mathrm{CH}$, HuTH, Lu SN, Kuo FY, Chen CH, Wang JH, Huang CM, Lee CM, Lin $\mathrm{CY}$, Yen $\mathrm{YH}$, et al. Circulating microRNAs as biomarkers for diagnosis of early hepatocellular carcinoma associated with hepatitis B virus. Int J Cancer. 2016;138(3):714-20.

37. Ke-Qin L. The effect of chemokine CC motif ligand 19 on the proliferation and migration of hepatocellular carcinoma. Tumour Biol. 2014. https:// doi.org/10.1007/s13277-014-2578-5.
38. Nielsen KO, Jacobsen KS, Mirza AH, Winther TN, Storling J, Glebe D, Pociot F, Hogh B. Hepatitis B virus upregulates host microRNAs that target apoptosis-regulatory genes in an in vitro cell model. Exp Cell Res. 2018;371(1):92-103.

39. Alkozai EM, Porte RJ, Adelmeijer J, Zanetto A, Simioni P, Senzolo M, Lisman T. Levels of angiogenic proteins in plasma and platelets are not different between patients with hepatitis B/C-related cirrhosis and patients with cirrhosis and hepatocellular carcinoma. Platelets. 2015;26(6):577-82.

\section{Publisher's Note}

Springer Nature remains neutral with regard to jurisdictional claims in published maps and institutional affiliations.
Ready to submit your research? Choose BMC and benefit from:

- fast, convenient online submission

- thorough peer review by experienced researchers in your field

- rapid publication on acceptance

- support for research data, including large and complex data types

- gold Open Access which fosters wider collaboration and increased citations

- maximum visibility for your research: over $100 \mathrm{M}$ website views per year

At BMC, research is always in progress.

Learn more biomedcentral.com/submissions 